\title{
On the Cover of this Issue: Relaxation and Recovery in Hydrogel Friction on Smooth Surfaces by B. Wu, J. S. Méndez Harper, and J. C. Burton
}
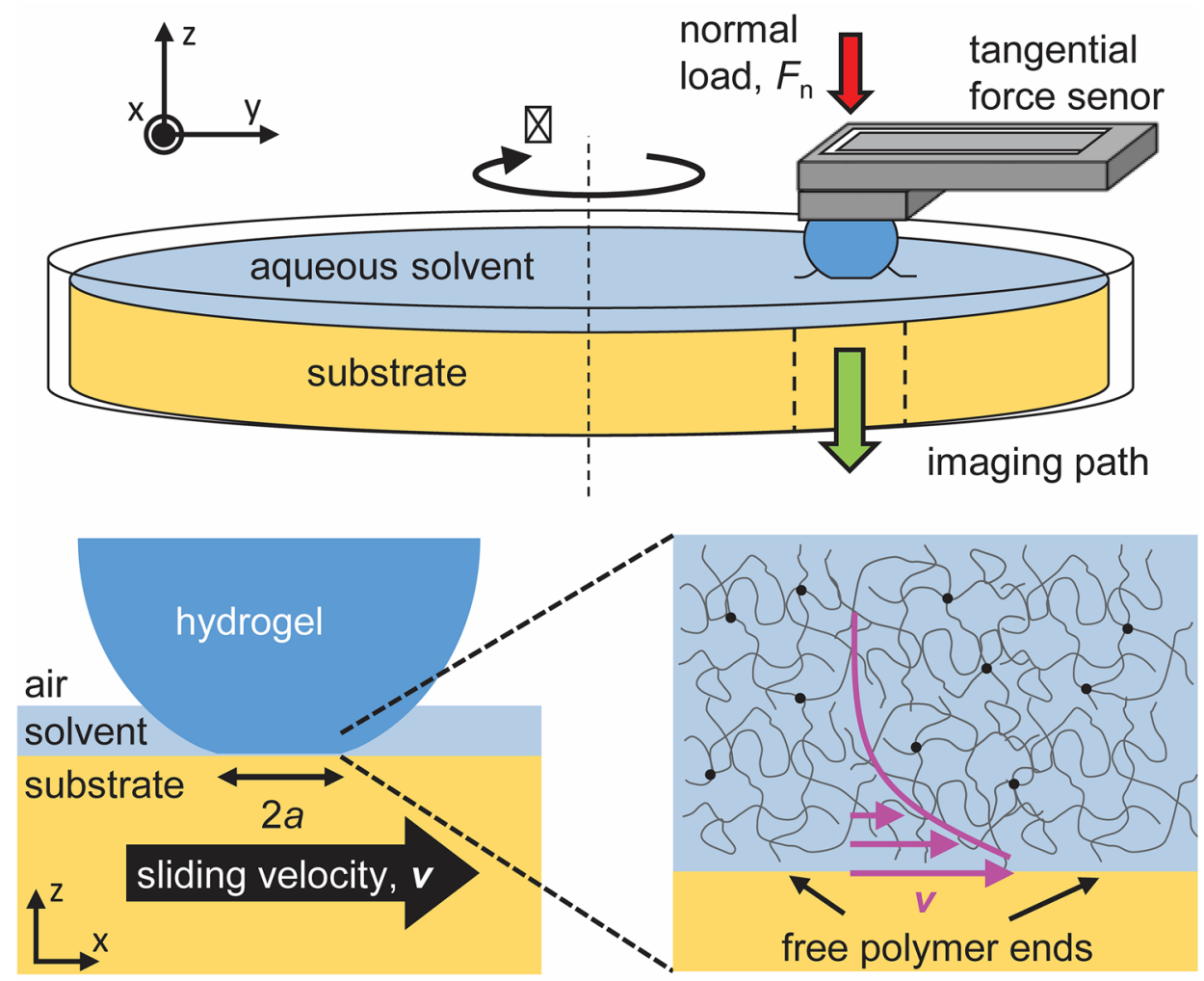

\section{Relaxation and Recovery in Hydrogel Friction on Smooth Surfaces} by B. Wu, J. S. Méndez Harper, and J. C. Burton 